\title{
Multilayer phosphor-in-glass packaging for the development in WLED color uniformity
}

\author{
Huu Phuc Dang', Phung Ton That ${ }^{2}$, Tri-Vien $\mathrm{Vu}^{3}$ \\ ${ }^{1}$ Faculty of Fundamental Science, Industrial University of Ho Chi Minh City, Vietnam \\ ${ }^{2}$ Faculty of Electronics Technology, Industrial University of Ho Chi Minh City, Vietnam \\ ${ }^{3}$ Modeling Evolutionary Algorithms Simulation and Artificial Intelligence, Faculty of Electrical and Electronics \\ Engineering, Ton Duc Thang University, Vietnam
}

\begin{tabular}{|c|c|}
\hline Article Info & ABSTRACT \\
\hline Article history: & \multirow{11}{*}{$\begin{array}{l}\text { When mentioning the remote phosphor structure, the most noticeable } \\
\text { advantage is its higher luminous flux than any other structure's. However, } \\
\text { there are existing flaws in their color uniformity and color rendering index } \\
\text { (CRI). Thus, the improvements in these two optic factors must be improved } \\
\text { for the better usage of remote phosphor geometry in modern WLED devices. } \\
\text { Many researchers have drawn their attention to this idea, and then it has } \\
\text { become the primary objective for their studies. In this paper, we also try to } \\
\text { accomplish the same result by adjusting the distances between the phosphor } \\
\text { layers and through that enhance the optical properties of WLEDs. The mie- } \\
\text { scattering theory is applied in our calculations to ensure the reliability and } \\
\text { accuracy of experimental results. In our research, with distance d=0.64 mm, } \\
\text { the luminous flux grew } 9.7 \% \text { in comparison to the original value. At } 0.84 \\
\text { mm, the distance d enhanced the color uniformity by two times. In the } \\
\text { meantime, the CRI remained static during the course of experiment. With } \\
\text { suitable application, these results can bring valuable contributions to the } \\
\text { development of next WELDs generation. }\end{array}$} \\
\hline Received Aug 25, 2020 & \\
\hline Revised Sep 30, 2020 & \\
\hline Accepted Oct 15, 2020 & \\
\hline Keywords: & \\
\hline Color uniformity & \\
\hline Lambert-Beer Law & \\
\hline Luminous flux & \\
\hline Mie-scattering thoery & \\
\hline Triple-layer phosphor & \\
\hline WLEDs & \\
\hline
\end{tabular}

This is an open access article under the CC BY-SA license.

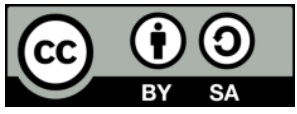

\section{Corresponding Author:}

Tri-Vien $\mathrm{Vu}$

Faculty of Electrical and Electronics Engineering

Ton Duc Thang University

No. 19 Nguyen Huu Tho Street, Tan Phong Ward, District 7, Ho Chi Minh City, Vietnam

Email: vutrivien@tdtu.edu.vn

\section{INTRODUCTION}

White light-emitting diodes (WLEDs) with remarkable lighting qualities such as long lifetime, power-saving, and high light efficiency has recently been employed more in the lighting industry and slowly replacing previous lighting methods. With the fast pace of WLED development, it is now suitable for in-door lighting, out-door lighting, automobile lights, and aesthetic illumination [1-3]. Previous enhancements in WLEDs were mostly achieved by perfecting III-nitride LEDs, which allows WLED devices to have better optical performance $[4,5]$. The improvements in III-nitride LEDs primarily focus on eliminating the charge separation occurs in the phosphor layer [6-9] and bettering the manufacturing of the ingredients [10-12]. The most cost effective and simplest procedure to produce WLEDs is integrating the chromatic lights of blue LEDs, a compound of adhesive gel, and yellow phosphor $\left(\mathrm{Y}_{3} \mathrm{Al}_{5} \mathrm{O}_{12}: \mathrm{Ce}^{3}\right)$. However, the WLED from this procedure is inadequate for high lighting applications because of its poor thermal performance even compared to others $[13,14]$. The cause for this incident is defined as the heat generation of phosphor, such 
level of thermal output reduces the light output and the wavelength deviation, moreover negatively effects the longevity of lighting devices $[15,16]$. One solution that was proposed to address this issue is changing the phosphor material to glass ceramic phosphors [17], ceramic phosphors [18], and phosphor-in-glass (PiG) $[19,20]$, which is well-known for great thermal endurance.

Along with the light output and the endurance of the devices, color quality is also the import which reflects through angular color uniformity (ACU) and the color rendering index (CRI) that have much attention in the lighting industry. Low color uniformity presents discomfort to the viewer's eyes, thus, enhancing the color uniformity is a priority in any research of WLED development for any packaging configuration [21]. One obvious method to reduce the deviation of color is to equalize the amount of phosphor distributed across LEDs chips. Sommer and associates have employed this theory and created a configuration with modified structure and phosphor compound to maximize the angular color uniformity (ACU) [22]. Another method that was proposed by Zheng et al. to enhance the ACU is using a substrate structure and coating the WLEDs by immersion [23]. Liu et al. project suggested that stacking the phosphor layers and applying inversed concentration distribution would lead to better color uniformity than the configuration with one phosphor layer. Although these methods achieved positive results for said subject, however, that their complexity and production expense are not optimal for serial production. Thus, the remote phosphor structure, which uses the principal of providing a calculated distance between the phosphor layers is a more practical approach. The mechanism of remote structure not only improves the light output of WLEDs but also increases the color quality by preventing the generated heat from stacking. The remote structure definitely an effective method to improve the optical properties of WLEDs, however, the angular color quality is not among the properties that remote structure benefits. To include the angular color quality improvement in the remote phosphor's advantages, many researchers have tried to adjust the structure organization such as Kuo et al. with a structure that has a blank circular area to strengthen the blue light extraction. Huang et al. balance the chromatic light distribution in WLEDs by introducing a patterned sapphire substrate that enhances the scattered blue light [24]. In another study, Chen et al. diffused the $\mathrm{ZrO}_{2}$ nanoparticles onto the package as a solution to stabilize the color discrepancy. The effectiveness of the mentioned solutions was expressed in WLEDs with silicone and phosphor compound. However, in the case of WLEDs with PiG, there have not been many researches focusing on how to improve the angular color uniformity in this type of lighting device [25]. For PiG WLEDs, the disproportion between the blue and yellow leads to bad color quality and an effect called the yellow ring that surrounds the projected light of WLEDs. Thus, finding a solution for color uniformity in WLEDs with PiG is an important target for WLEDs development.

Controlling the appearance of $\mathrm{PiG}$ is an essential criterion to improve color uniformity, however, the complicated procedure to create PiG makes it hard to achieve this goal. Therefore, we demonstrate a more effective manufacturing method of patterned PiG that allows better color uniformity to be obtained. Through accurate calculations on subject's parameters and simulation results, we have verified that cone-shaped PiG WLEDs with multiple layers produce more blue lights at wider angles and better color uniformity.

\section{RESEARCH METHOD}

The simulation program used to measure the optical properties of WLEDs at $5600 \mathrm{~K}$ and $8500 \mathrm{~K}$ in this research is LightTools. The results were later put together to study the impacts that different alterations had on WLED lighting performance. The simulated model of a WLED consists of blue LED chips, multiple phosphor layers, one reflector cup, and a silicone layer. The shape of the model is half-dorm that covers the content underneath, as can be seen in Figure 1 (a).

The measurements for the reflector cup of WLEDs model are $2.07 \mathrm{~mm}$ high, $8 \mathrm{~mm}$ long for the bottom surface. The LED blue chips attached to the reflector own the size of $1.14 \mathrm{~mm}$ width and $0.15 \mathrm{~mm}$ height and emit 1.16W at $453 \mathrm{~nm}$ peak wavelength, as shown in Figure 1 (b). The chips are ensured to be identical to obtain the most accurate results from light emissions. In addition, the $0.08 \mathrm{~mm}$ phosphor layer is laid over the chips, see Figure 1 (c) and Figure 1 (d). Throughout the course of experiments, the distance between the phosphor layers is changed several times, and the results are compared to define their effects on the optical properties of WLEDs. The phosphor particles are in spherical form and approximately $14.5 \mu \mathrm{m}$. In the beginning, the distance between the phosphor layer in WLEDs configuration is $0.24 \mathrm{~mm}$ and constantly raised to $0.84 \mathrm{~mm}$. The adjustment will impact the outcomes of light output and color uniformity, which leads us to the optimal distance for WLEDs' performance. In the experiment, it is crucial to keep the CCT index stable so that the results can be consistent, and to achieve this the phosphor concentration is adjusted in response to each distinct distance, which can be seen from Figure 2. 


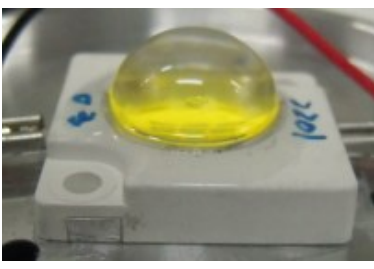

(a)

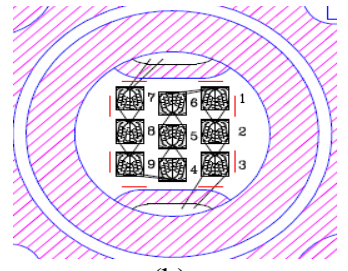

(b)

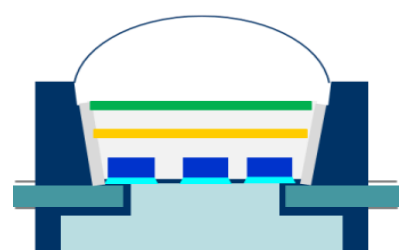

(c)

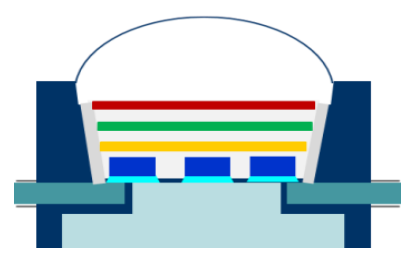

(d)

Figure 1. Photograph of WLEDs structure; (a) actual WLEDs, (b) bonding diagram, (c) illustration of dual-layer phosphor structure, and (d) illustration of tripple-layer phosphor structure

In Figure 2, the phosphor concentration and particle size in the red phosphor layer and green phosphor layer are settled while those values in yellow phosphor layer is altered. Comparing to the in-cup phosphor and conformal phosphor, the color rendering index in remote phosphor structure is much harder to accomplish. Therefore, the idea of adding more chromatic phosphor layer to WLEDs for better optical values such as red phosphor for color rendering index, green phosphor for luminous flux and color uniformity, should be considered. To demonstrate the efficiency of these extra layers, this study measured their performance in 4 different WLEDs with color temperature ranging from $5600 \mathrm{~K}$ to $8500 \mathrm{~K}$.

In all the researches mentioned above, the studied targets are single chip WLEDs with color temperature lower than $7000 \mathrm{~K}$. However, the computation of lighting performance in WLEDs with color temperature above $7000 \mathrm{~K}$ is more challenging due to the impacts from other factors such as light scattering and light absorption from phosphor. In higher color temperature WLEDs, the difference in phosphor concentration becomes more obvious. The higher color temperature, the lower yellow phosphor concentration. These changes in distance and phosphor concentration are impactful to the luminous flux and color quality. Not to mention, the red phosphor layer and green phosphor layer also have great influences over the development of optical properties. The detailed measurements of these influences are demonstrated in section 3 .

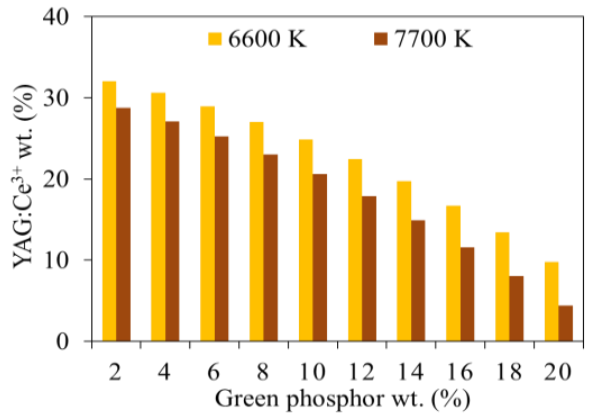

(a)

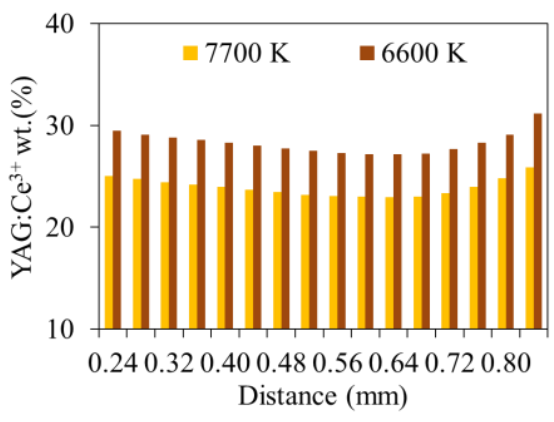

(b)

Figure 2. The concentration of yellow YAG: $\mathrm{Ce}^{3+}$ phosphor as a function of $\mathrm{d}$ and green phosphor wt;

(a) dual-layer phosphor structure, and (b) tripple-layer phosphor structure

\section{RESULTS AND ANALYSIS}

Figure 3 shows the changes that distance d brings to the lumen output of WLEDs. As observed, when $\mathrm{d}$ increases from $0.24 \mathrm{~mm}$ to $0.64 \mathrm{~mm}$, the lumen output tends to increase and reaches the highest value of $968 \mathrm{~lm}$ at $\mathrm{d}=0.64 \mathrm{~mm}$. The explanation for this result can be presented as the following point. When the distance expands, it is easier for the light transmission between the layers to operate, thus, the light output increases. On the other hand, for distance above $0.64 \mathrm{~mm}$, the yellow phosphor YAG: $\mathrm{Ce}^{3+}$ also rises, which leads to an opposite effect and reduces the light output. The discrepancies between light output at different distances $\mathrm{d}$ can be tracked from Table 1 .

The increase in light output is the result of two events: first, the presence of the green phosphor in WLEDs and second, the appropriate distance $d$ leads to the optimization of light transmission. When $\mathrm{d}=0.64 \mathrm{~mm}$, the lumen output is increased, which is $71 \mathrm{~lm}$ higher than the result of the original distance of $0.24 \mathrm{~mm}$. This has confirmed that the luminous flux can achieve $9.7 \%$ enhancement from the original value. 
Table 1. The luminous flux as a function of $\mathrm{d}$ with the average CCTs

\begin{tabular}{cccc}
\hline CCTs & $\mathbf{0 . 2 4} \mathbf{~ m m}$ & $\mathbf{0 . 6 4} \mathbf{~ m m}$ & $\mathbf{0 . 8 4} \mathbf{~ m m}$ \\
\hline $6600 \mathrm{~K}$ & $730 \mathrm{~lm}$ & $801 \mathrm{~lm}$ & $627 \mathrm{~lm}$ \\
$7700 \mathrm{~K}$ & $850 \mathrm{~lm}$ & $917 \mathrm{~lm}$ & $729 \mathrm{~lm}$ \\
\hline
\end{tabular}

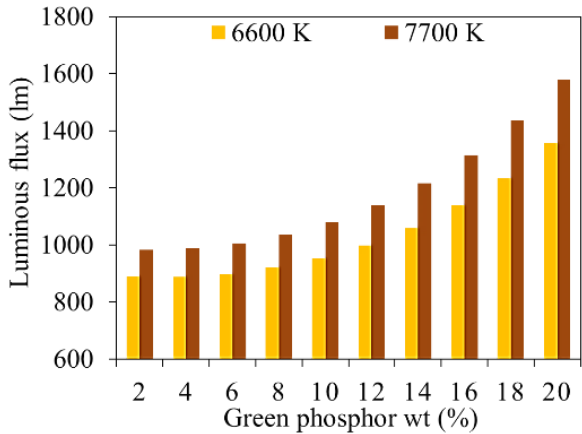

(a)

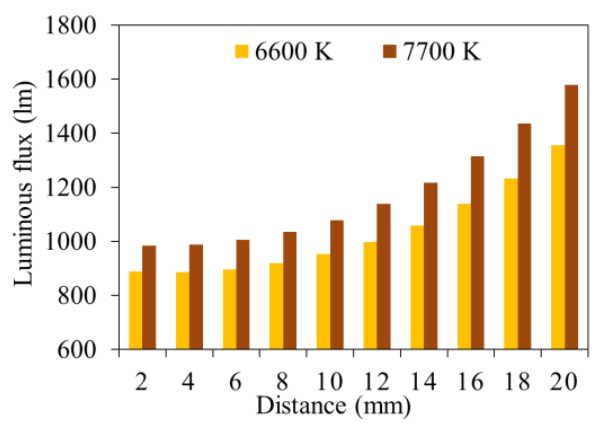

(b)

Figure 3. The luminous output of WLEDs as a function of $d$ and green phosphor wt;

(a) dual-layer phosphor structure, and (b) tripple-layer phosphor structure

The advantages of modifying distance $d$ are the enhancement in not only the light output but also the color quality, which are attained by reducing the color deviation, as presented Figure 4 . When expanding the distance $\mathrm{d}$, aside from allowing the emitted light to move more freely among the phosphor layer, it also leads to better scattering events as a result of wider scattering space. The color deviation according to each distance point is presented Table 2. As can be seen from the content of Table 2, the color deviation at $8500 \mathrm{~K}$ has the most noticeable improvement. The color deviation drops nearly $50 \%$, which means the color uniformity of WLEDs is two times better.

The lower color deviation means the better color uniformity and color quality, so one of the targets of this research is achieved. At all correlated color temperature, the lowest color deviation is always obtained at $\mathrm{d}=0.84 \mathrm{~mm}$. The explanations for this relation between the color deviation and correlated color temperature are the appearances of red phosphor and green phosphor in the configuration and the increased scattering space. The expansion in scattering space, which is the primary reason for color deviation reduction, strengthens the fusion between the chromatic lights, thus, leading to better color quality. Therefore, it can be confirmed that providing a suitable distance between the phosphor layers is the optimal solution for better luminous flux and color uniformity in WLEDs.

Table 2. The color deviation as a function of $d$ with the average CCTs

\begin{tabular}{cccc}
\hline CCTs & $0.24 \mathrm{~mm}$ & $0.64 \mathrm{~mm}$ & $0.84 \mathrm{~mm}$ \\
\hline $6600 \mathrm{~K}$ & $2803 \mathrm{~K}$ & $1762 \mathrm{~K}$ & $1939 \mathrm{~K}$ \\
$7700 \mathrm{~K}$ & $3872 \mathrm{~K}$ & $2625 \mathrm{~K}$ & $2275 \mathrm{~K}$ \\
\hline
\end{tabular}

The results of color rendering index (CRI) are demonstrated in Figure 5. As the graphs in Figure 5 suggest, the rendering ability will remain static despite the fluctuation in distance $\mathrm{d}$. This event brings up the question about the influence of red phosphor in this case, as the usual reaction when adding red phosphor would be the CRI rises while the luminous flux falls. However, an intriguing result occurs in this particular situation that the CRI was unchanged while luminous flux and color uniformity were enhanced. This traces back to the arrangement of the phosphor layers within the configuration, in which the red phosphor layer is placed at the top. With this kind of arrangement, the emitted light after passing through the green phosphor layer has mostly transformed into the white light before reaching the red phosphor layer. Considering the low white light absorption of red phosphor, it explained why the red phosphor is not that effective in improving the color rendering index as intended. However, adding the red phosphor layer is still beneficial because of the light scattering and color uniformity enhancement. Although the CRI does not receive any development, the higher luminous flux and color quality are already noteworthy results for WLEDs development. 


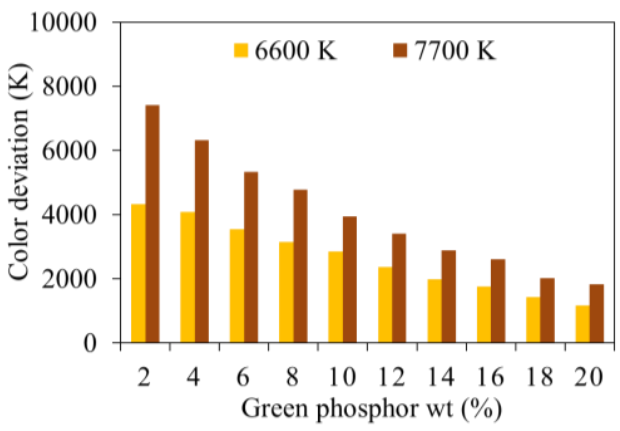

(a)

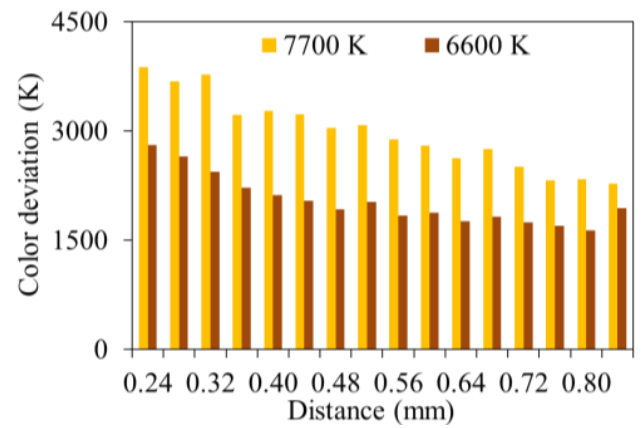

(b)

Figure 4. The color deviation value of WLEDs as a function of $\mathrm{d}$ and green phosphor wt; (a) dual-layer phosphor structure, and (b) tripple-layer phosphor structure

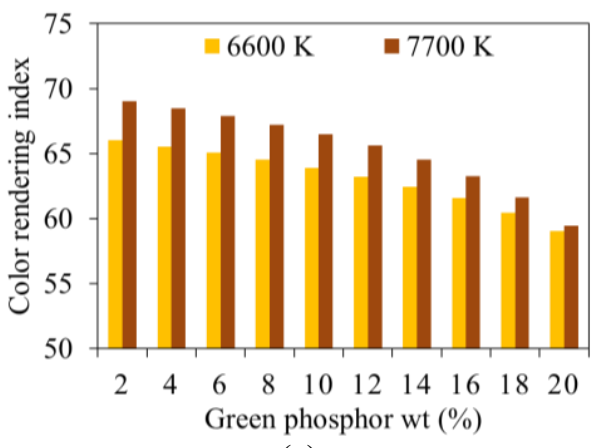

(a)

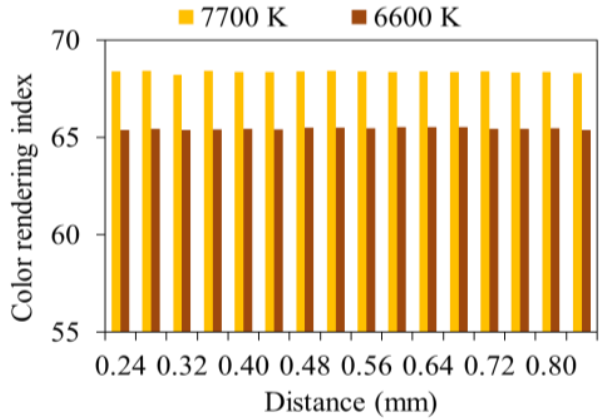

(b)

Figure 5. The color rendering index of WLEDs as a function of $\mathrm{d}$ and green phosphor wt; (a) dual-layer phosphor structure, and (b) tripple-layer phosphor structure

\section{CONCLUSION}

In this manuscript, the influences of distance $d$ on WLEDs optical properties are demonstrated in details. Through the application of Mie-scattering theory, we have computed and verified the results of optical simulation. In particular, luminous flux achieved $9.7 \%$ growth at $d=0.64$ in comparison to $d=0.24$. However, higher distance (above $0.64 \mathrm{~mm}$ ) will result in a minimal decrease in luminous flux, because YAG: $\mathrm{Ce}^{3+}$ concentration rises and obstructs the light transmission. Therefore, for optimal lighting performance, the manufacturer should choose $\mathrm{d}=0.64 \mathrm{~mm}$. Beside the luminous flux effects, the colour uniformity is also considered for development in distance application. According to the experimental results, the increase in distance can effectively reduce the color deviation by $50 \%$, which means at $\mathrm{d}=0.84$, color uniformity is two times enhanced for WLEDs at $8500 \mathrm{~K}$. This distance can be applied by manufacturers who demand higher color quality in their products. The final performance indicator, color rendering index (CRI), seems to be unaffected by the fluctuation of the distance between layers. The explanation for this event is because the red phosphor layer at the top has the low white light absorption capacity, which makes the red phosphor layer less impactful to the rendering ability of WLEDs, leading to no significant change in CRI when the distance increases. On the other hand, the red phosphor layer is effective in strengthening the scattering events in the configuration and stabilizing the CRI. In conclusion, with a proper usage of the information given in this research, manufacturers can create better WLEDs that fit their targets.

\section{REFERENCES}

[1] C. Han et al., "Effect of surface recombination in high performance white-light $\mathrm{CH}_{3} \mathrm{NH}_{3} \mathrm{PbI}_{3}$ single crystal photodetectors," Opt. Express, vol. 26, pp. 26307-26316, 2018.

[2] N. T. Canh et al., "Electrohydrodynamic jet-sprayed quantum dots for solution-processed light-emitting-diodes," Opt. Mater. Express, vol. 8, pp. 3738-3747, 2018.

[3] C. Huang et al., "Bandwidth correction of spectral measurement based on Levenberg-Marquardt algorithm with improved Tikhonov regularization," Appl. Opt, vol. 58, pp. 2166-2173, 2019. 
[4] Y. Xie et al., "Encapsulated room-temperature synthesized $\mathrm{CsPbX} 3$ perovskite quantum dots with high stability and wide color gamut for display," Opt. Mater. Express, vol. 8, pp. 3494-3505, 2018.

[5] T. Kozacki et al., "Fourier rainbow holography," Opt. Express, vol. 26, pp. 25086-25097, 2018.

[6] B. Xu et al., "Time-multiplexed stereoscopic display with a quantum dot-polymer scanning backlight," Appl. Opt, vol. 58, pp. 4526-4532, 2019

[7] J. Zhang et al., "Ce ${ }^{3+} / \mathrm{Mn}^{2+}$-activated $\mathrm{Ca} 7, \mathrm{PO}_{4.2}, \mathrm{SiO}_{4.2}$ : efficient luminescent materials for multifunctional applications," Opt. Express, vol. 26, pp. A904-A914, 2018.

[8] S. Jost et al., "CIE 2017 color fidelity index Rf: a better index to predict perceived color difference?," J. Opt. Soc. Am. A, vol. 35, pp. B202-B213, 2018.

[9] B. G. Assefa et al., "Realizing freeform lenses using an optics 3D-printer for industrial based tailored irradiance distribution," OSA Continuum, vol. 2, pp. 690-702, 2019.

[10] S. Cincotta et al., "High angular resolution visible light positioning using a quadrant photodiode angular diversity aperture receiver, QADA.," Opt. Express, vol. 26, pp. 9230-9242, 2018.

[11] A Correia et al., "Holistic opto-thermal simulation framework for high-brightness light sources based on fluorescent conversion," Opt. Express, vol. 27, pp. A1324-A1337, 2019.

[12] W. Wang et al., "On the development of an effective image acquisition system for diamond quality grading," Appl. Opt, vol. 57, pp. 9887-9897, 2018.

[13] T. P. White et al., "Feature issue introduction: halide perovskites for optoelectronics," Opt. Mater. Express, vol. 8, pp. 231-234, 2018.

[14] T. P. White et al., "Feature issue introduction: halide perovskites for optoelectronics," Opt. Express, vol. 26, pp. A153-A156, 2018.

[15] M. C. Aguilar et al., "Automated instrument designed to determine visual photosensitivity thresholds," Biomed. Opt. Express, vol. 9, pp. 5583-5596, 2018.

[16] C. S. Kim et al., "High-speed color three-dimensional measurement based on parallel confocal detection with a focus tunable lens," Opt. Express, vol. 27, pp. 28466-28479, 2019.

[17] A. A. Kaminari et al., "Linking Infrared Spectra of Laboratory Iron Gall Inks Based on Traditional Recipes with their Material Components," Appl. Spectros, vol. 72, pp. 1511-1527, 2018.

[18] Q. T. Vinh et al., "Preliminary measure for the characterization of the usefulness of light sources," Opt. Express, vol. 26, pp. 14538-14551, 2018.

[19] Y. P. Chang et al., "New scheme of LiDAR-embedded smart laser headlight for autonomous vehicles," Opt. Express, vol. 27, pp. A1481-A1489, 2019.

[20] X. Li et al., "Projection lithography patterned high-resolution quantum dots/thiol-ene photo-polymer pixels for color down conversion," Opt. Express, vol. 27, pp. 30864-30874, 2019.

[21] M. Dupont-Nivet, C. I. Westbrook, and S. Schwartz, "The role of trap symmetry in an atom-chip interferometer above the Bose-Einstein condensation threshold," in 2019 Conference on Lasers and Electro-Optics Europe and European Quantum Electronics Conference, OSA Technical Digest, Optical Society of America, 2019.

[22] S. Rasouli et al., "Colorful radial Talbot carpet at the transverse plane," Opt. Express, vol. 27, pp. 17435-17448, 2019.

[23] S. P. Groth et al., "Circulant preconditioning in the volume integral equation method for silicon photonics," J. Opt. Soc. Am. A, vol. 36, pp. 1079-1088, 2019.

[24] Q. Zhang et al., "Excellent luminous efficiency and high thermal stability of glass-in-LuAG ceramic for laserdiode-pumped green-emitting phosphor," Opt. Lett, vol. 43, pp. 3566-3569, 2018.

[25] Y. Hu et al., "Greatly enhanced persistent luminescence of $\mathrm{YPO}_{4}: \mathrm{Sm}^{3+}$ phosphors via $\mathrm{Tb}^{3+}$ incorporation for in vivo imaging," Opt. Express, vol. 28, pp. 2649-2660, 2020. 